\title{
A Test of the Tanaka Model with NOAA 10488
}

\author{
Hui Zhao ${ }^{1}$, Yuzong Zhang ${ }^{1,2}$, Jie Jiang ${ }^{1}$ and Jingxiu Wang ${ }^{1}$ \\ ${ }^{1}$ National Astronomical Observatories, Chinese Academy of Science, Beijing 100012, China \\ email: zhaohui@ourstar.bao.ac.cn \\ ${ }^{2}$ Department of Astronomy, Beijing Normal University, Beijing 100875, China
}

\begin{abstract}
We investigated the complex subsurface magnetic rope structure of a super-active region NOAA 10488. With the set of twisted magnetic loop, knot and bifurcate configuration, we could explain the complicated flux emerging, developing and disappearing by following Tanaka model (Tanaka, 1991). Based on Huairou photospheric vector magnetograms, we calculated the current helicity and found the dominant helicity sign is positive. We deduced that the whole active region might be one twisted magnetic rope.
\end{abstract}

Keywords. Sun: magnetic fields, sunspots

\section{Introduction}

The details and origin of magnetic rope structure under the photosphere are still not known clearly. In 1991 Tanaka firstly sought complex magnetic rope structures under the surface as inferred from detailed evolution data and analyzed the August 1972 region successfully. Kurokawa, Wang, \& Ishii (2002) constructed a model of an emerging twisted flux rope to explain the outstanding features of the sunspots evolution in NOAA AR9026 (Figure 2(a)). Fan et al. (1999) performed three-dimensional MHD simulations of the rise of twisted magnetic flux tubes in an adiabatically stratified model solar convection zone. In this paper we try to use a set of the complex subsurface magnetic rope structure to explain NOAA AR10488's emerging, evolving and disappearing and test the model of Tanaka.

\section{Data Analysis}

During two weeks in late October and early November 2003, there is a series of large solar activities. The active region NOAA 10488 emerged on October 26 and developed into a large AR rapidly on October 27, then stepped into its phase of decay when approaching the west edge of the sun on November 3. With the MDI longitudinal magnetograms we traced its continuous evolution. Hence we can pick up pairs of bipolar appearance in the same time and identify the topological connectivity of magnetic field lines. And the sub-photospheric structures can also be identified through the movement, rotation and decay of longitudinal magnetic field.

By photospheric vector magnetograms and images of $\mathrm{H} \alpha$ we obtain more details of transverse field alignments. The 180 degree ambiguity in the observed field azimuth was carefully corrected by both theoretical and empirical methods (Wang, 1996). We calculated current helicity based on the vector field and found the dominant sign of helicity is positive (Figure 1(b)). So we could consider the whole active region may be one twisted magnetic rope like the model of Tanaka. The Figure 2(b) is one of possible structures which satisfy the evolvement of NOAA AR 10488. 


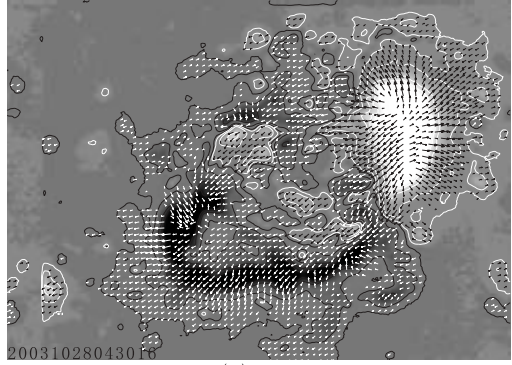

(A)

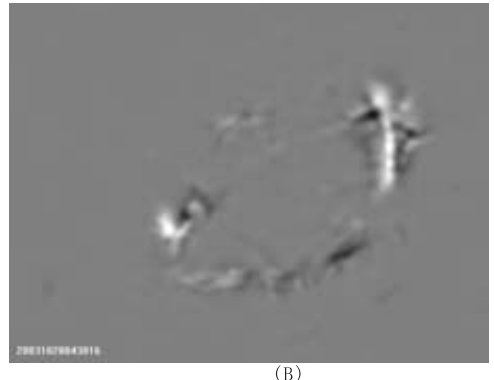

(B)

Figure 1. (a) One of the photospheric vector magnetograms from Huairou (which corresponding to the second longitudinal magnetogram employed in Fig. 3.). (b) The current helicity for (a).
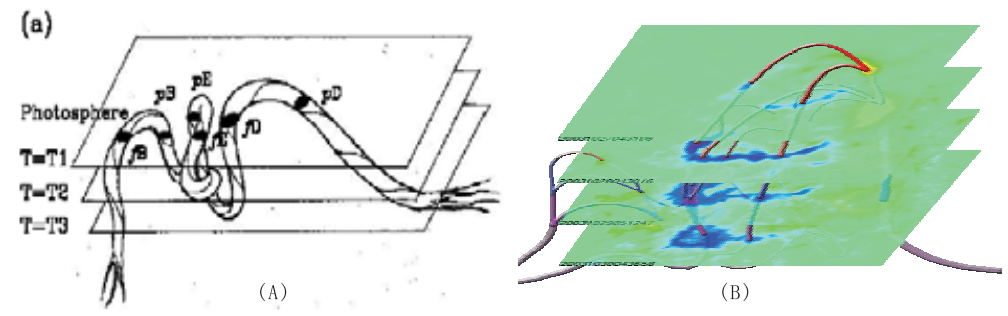

Figure 2. (a) Schematic drawing of an emerging twisted flux rope. The photospheric surfaces are drawn at three successive times(Kurokawa, Wang, \& Ishii (2002)). (b) The schematic drawing of an emerging twisted flux rope. The background is the Huairou's longitudinal magnetograms at the four close time with (a). The sections of the flux rope cut by each longitudinal magnetogram are the locations of sunspots of that time.

\section{Conclusion and Discussion}

Tanaka model can reasonably explain the emerging and disappearing of sunspots and corresponding phenomena. However, to a given active region, the structure of flux rope drawn only by this model is not unique. The calculation of the current helicity distribution of NOAA AR 9026 basically accords with Kurokawa's flux rope structure of the same region. In our work the loop and hook twist structure, the bifurcate configuration and their evolution with time could explain the increasing, decreasing and disappearing of the sunspots, with the twist structure of knot, it is easy to understand the magnetic shearing. In addition, we try to model the complex magnetic configuration of the AR 10488 following the minimum energy principle.

\section{Acknowledgements}

The work is supported by the National Natural Science Foundation of China(10233050) and the National Key Basic Science Foundation(TG2000078404).

\section{References}

Hiroki Kurokawa, Tongjiang Wang, \& Takako T. Ishii 2002, ApJ 572, 598-608

Katsuo Tanaka 1991, Solar Physics 136, 133-149

Y.Fan, E. G., Zweibel, M. G. Linton, \& Fisher, G. H. 1999, ApJ 521, 460-477 